\title{
Blue Thermoluminescence Emission Of Annealed Lithium Rich Aluminosilicates
}

\author{
V. Correcher* and Y. Rodriguez-Lazcano \\ CIEMAT. Av. Complutense 22, Madrid 28040, Spain \\ J. Garcia-Guinea and E. Crespo-Feo \\ Museo Nacional de Ciencias Naturales, C/José Gutierrez Abascal 2, Madrid 28006, Spain \\ (Received on 28 April, 2010)
}

\begin{abstract}
The blue thermoluminescence (TL) emission of different thermally annealed (i) $\beta$-eucryptite $\left(\mathrm{LiAlSiO}_{4}\right)$, (ii) virgilite-petalite $\left(\mathrm{LiAlSi}_{5} \mathrm{O}_{12}\right)$ and (iii) virgilite-petalite-bikitaite $\left(\mathrm{LiAlSi}_{10} \mathrm{O}_{22}\right)$ mixed crystals have been studied. The observed changes in the TL glow curves could be linked to simultaneous processes taking place in the lithium aluminosilicate lattice structure (phase transitions, consecutive breaking linking of bonds, alkali self-diffusion, redox reactions, etc). The stability of the TL signal after four months of storage performed at RT under red light, shows big differences between annealed $\left(12\right.$ hours at $\left.1200^{\circ} \mathrm{C}\right)$ and non-annealed samples. The fading process in non-annealed samples can be fitted to a first-order decay mathematical expression; however preheated samples could not be reasonably fitted due to the highly dispersion detected. The changes observed in the X-ray diffractograms are in the intensity of the peaks that denote modifications in the degree of crystallinity and, in addition, there are some differences in the appeareance of new peaks that could suppose new phases (e.g. $\beta$-spodumene).
\end{abstract}

Keywords: Phosphors; thermoluminescence; aluminosilicates

\section{INTRODUCTION}

The thermal stability of the phases of the LAS $\left(\mathrm{LiO}_{2}-\right.$ $\left.\mathrm{Al}_{2} \mathrm{O}_{3}-\mathrm{SiO}_{2}\right)$ system has led these materials to be employed in different applications (i.e. turbine engine heat exchangers, mirror substrates for telescopes, cookware, etc.)[1]. However, the optical properties in general and the luminescence emission in particular of different members of LAS series have been scarcely investigated. These materials are phosphors with many different chemical compositions that could be potentially used to measure the radiation absorbed dose based on the TL emission. The ionising radiation absorbed by the phosphor at room temperature gives rise to freed electrons that become trapped in the lattice defects of the material (point defects, vacancies, Schottky defects, Frenkel defects, dislocations, planar defects, etc.) It is accepted that in this process such electrons will be trapped for long periods while the material is stored at room temperature. When the temperature is increased, the electrons can release from the traps and in a recombination process they reach more stable energies with emission of photons [2,3]. What is required for a suitable TLD material, among others, is: (i) high TL intensity, (ii) high stability of the TL signal during the storage of the material (i.e. low fading), (iii) high linearity of the TL emission with the dose in the range of interest, (iv) the trap distribution must be as simple as possible, (v) the TL emission must be in the range of UV-IR and (vi) the material must be thermally and chemically stable. Some of the features of the blue emission of these LAS materials have been previously analysed in our laboratory to determine their potential use as dosemeters [4-7], however there are no studies on the effect of strong thermal annealing on the blue emission of such materials. This paper is basically focused on the study of the stability of the blue TL signal (at 400nm) of some annealed and non-annealed members of the LAS sys-

${ }^{*}$ Electronic address: v.correchereciemat.es tem $\left(\mathrm{LiAlSiO}_{4}, \mathrm{LiAlSi}_{5} \mathrm{O}_{12}\right.$ and $\left.\mathrm{LiAlSi}_{10} \mathrm{O}_{22}\right)$ with the time of storage at room temperature (RT), up to four months. The decay of the TL signal, or fading, could be defined as the spontaneous loss of signal of the previously irradiated material with the time that is mainly caused by thermal effect and relaxation of the lattice after receiving the ionising radiation. Thus, in the thermal fading process, the electrons hosted in lower trap energy will fade faster than the more energetic ones caused by their higher probability of transition. The awareness of the evolution of the fading effect is of great interest as a starting point to determine the dosimetric possibilities of these materials.

\section{MATERIALS AND METHODS}

Lithium-aluminium stuffed derivatives of silica polymorphs, $\quad \beta$-eucryptite $\left(\mathrm{LiAlSiO}_{4}\right), \quad$ virgilite-petalite $\left(\mathrm{LiAlSi}_{5} \mathrm{O}_{12}\right)$ and virgilite-petalite-bikitaite $\left(\mathrm{LiAlSi}_{10} \mathrm{O}_{22}\right)$ mixed crystals, were synthesised using Hamilton and Henderson's gelling method [8]. The elements $(\mathrm{Li}, \mathrm{Al}, \mathrm{Si})$ were introduced from precursor powders, in three steps: i) Lithium from $\mathrm{Li}_{2} \mathrm{CO}_{3}$, ii) Silica as TEOS which is tetraethylorthosilicate $\left(\mathrm{Si}_{2} \mathrm{C}_{2} \mathrm{H}_{5} \mathrm{O}_{4}\right)$ and iii) Aluminium as crystals of $\mathrm{Al}\left(\mathrm{NO}_{3}\right)_{3} \cdot 9 \mathrm{H}_{2} \mathrm{O}$. $\mathrm{Al}$ and $\mathrm{Li}$ cations were added in stoichiometric proportions to preserve the electrical neutrality of the quartz lattice $\left(A l^{3+}+\mathrm{Li}^{+}=S i^{4+}\right)$. The structural data of the samples were measured by a Siemens D-5000 X-ray automated diffractometer (XRD) using the $\mathrm{CuK} K_{\alpha}$ radiation with a $N i$ filter at a setting of $40 \mathrm{kV}$ and $200 \mathrm{~mA}$. Patterns were obtained by step scanning from $2^{\circ}$ to $64^{\circ} 2 \theta$ in steps of $0.020^{\circ}$ counting time of $6 s$ per step. The resultant XRD profiles of the powdered lithium aluminosilicate samples were studied by XPOWDER software performing background subtraction, $K \alpha_{2}$ stripping and chemical elements restrained to $\mathrm{Al}-\mathrm{Si}-\mathrm{Li}$ [9].

Thermoluminescence (TL) measurements were carried out using an automated Ris $\varnothing$ TL system model TL DA-12 [10]. This reader is provided with an EMI 9635 QA photo- 
multiplier and the emission was observed through a blue filter (a FIB002 of the Melles-Griot Company) where the wavelength is peaked at $320-480 \mathrm{~nm}$; FWHM is $80 \pm 16 \mathrm{~nm}$ and peak transmittance (minimum) is $60 \%$. It is also provided with a ${ }^{90} \mathrm{Sr} /{ }^{90} \mathrm{Y}$ source with a dose rate of $0.020 \mathrm{~Gy} \mathrm{~s}{ }^{-1}$ calibrated against a ${ }^{60} \mathrm{Co}$ photon source in a secondary standards laboratory [11]. All the TL measurements were performed using a linear heating rate of $5^{\circ} \mathrm{C} \cdot \mathrm{s}^{-1}$ from RT up to $550^{\circ} \mathrm{C}$ in a $N_{2}$ atmosphere. Four aliquots of $5.0 \pm 0.1 \mathrm{mg}$ each of $\mathrm{LiAlSiO}_{4}, \mathrm{LiAlSi}_{5} \mathrm{O}_{12}$ and $\mathrm{LiAlSi}_{10} \mathrm{O}_{22}$ were used for each measurement. The samples were carefully powdered with an agate pestle and mortar to avoid triboluminescence [12] and sieved to obtain a grain size fraction under $50 \mu \mathrm{m}$. The incandescent background was subtracted from the TL data. Sample processing and measurements were made under red light to avoid the release of the trapped electrons from the semistable sites into hole centres (including luminescence centres) due to light sensitivity. The samples were preheated at $1200^{\circ} \mathrm{C}$ for $12 \mathrm{~h}$ in a programmable temperature controlled tubular oven with quartz chamber in open air. The heating rate to reach $1200^{\circ} \mathrm{C}$ was $100^{\circ} \mathrm{C} \cdot \mathrm{min}^{-1}$, and the annealed samples were cooled to RT at $4^{\circ} \mathrm{C} / \mathrm{min}$.

\section{RESULTS AND DISCUSSION}

\subsection{XRD characterization}

Figure 1 displays the powder XRD patterns of the three non-annealed (left-hand column) and annealed $1200^{\circ} \mathrm{C}$ for $12 \mathrm{~h}$ (right-hand column) stuffed derivatives of $\mathrm{LiAlSiO}_{4}$, $\mathrm{LiAlSi}_{5} \mathrm{O}_{12}$ and $\mathrm{LiAlSi}_{10} \mathrm{O}_{22}$. The strength of the thermal treatment induces (i) the creation of glassy phases that affects to the X-ray diffraction peaks giving rise to smaller intensities that supposes a lower degree of crystallinity and (ii) the appeareance of new maxima that suppose the presence of new phases such as $\beta$-spodumene since is a typical phase appearing when $\beta$-eucryptite is heated at temperatures over $1000^{\circ} C$ [13]. In fact, the initial settings focus the Boolean search-matching on the ICDD-PDF2 and RRUFF databases suggest the following card files: (i) $\mathrm{LiAlSiO}_{4}$ showing 21503 card (petalite) and cards 17-0533, 15-27 and 12-451 ( $\beta$-eucryptite) (Fig 1a); (ii) $\mathrm{LiAlSi}_{5} \mathrm{O}_{12}$ being mixtures of cards 31-707 (virgilite) and 21-503 (petalite) (Fig 1c) and (iii) $\mathrm{LiAlSi}_{10} \mathrm{O}_{22}$ being also mixtures of cards 31-707 (virgilite) and 21-503 (petalite) and additional minor amount of Card 75-755 (bikitaite) (Fig 1e). Conversely, these aliquots $\mathrm{LiAlSiO}_{4}, \mathrm{LiAlSi}_{5} \mathrm{O}_{12}$, and $\mathrm{LiAlSi}_{10} \mathrm{O}_{22}$ after the annealing at $1200^{\circ} \mathrm{C}$ for $12 \mathrm{~h}$ match with card $21-503$ ( $\beta$-spodumene; $\mathrm{Li}_{2} \mathrm{O} \cdot \mathrm{Al}_{2} \mathrm{O}_{3} \cdot 4 \mathrm{SiO}_{2}$ ) (Fig $1 \mathrm{~b}, \mathrm{~d}$ and e). We could also observe as these high temperature aliquots, under RT conditions, alter to zeolite bikitaite $\left(\mathrm{Li}_{2} \mathrm{Al}_{2} \mathrm{Si}_{4} \mathrm{O}_{12} .2 \mathrm{H}_{2} \mathrm{O}\right)$ by environmental hydration mechanisms.

\subsection{Thermoluminescence emission}

The TL glow curves of the three different 5Gy beta irradiated Li-rich aluminosilicates $\left(\mathrm{LiAlSiO}_{4}, \mathrm{LiAlSi}_{5} \mathrm{O}_{12}\right.$ and $\mathrm{LiAlSi}_{10} \mathrm{O}_{22}$ ) are displayed in figure 2 (left-hand plots cor- (a)

(b)
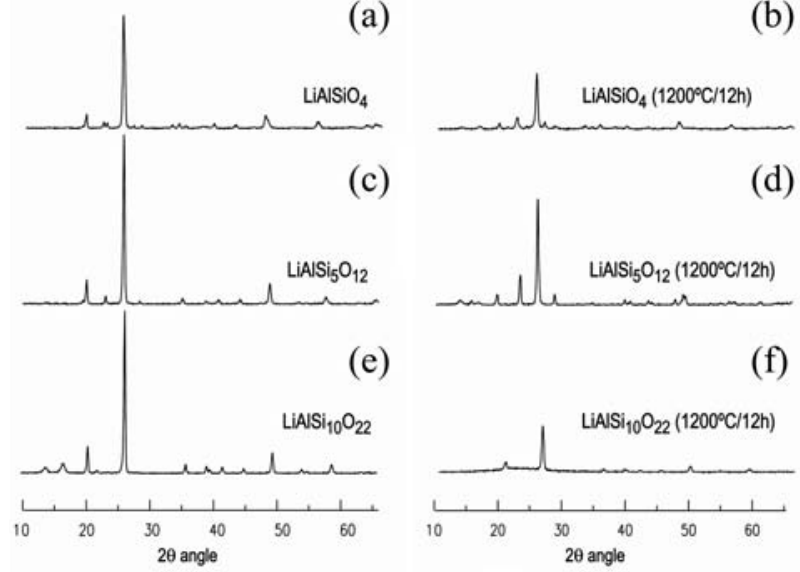

(e)

(f)

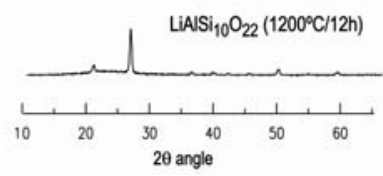

FIG. 1: X-ray diffraction patterns from the synthetic (a,c and e) non-annealed lithium-rich aluminosilicates and (b, $d$ and f) $12 \mathrm{~h}$ at $1200^{\circ} \mathrm{C}$ thermally annealed samples.

respond to non annealed samples and right-hand plots belong to $1200^{\circ} \mathrm{C}$ for 12 hours annealed material). All of the analysed Li-rich aluminosilicates $\left(\mathrm{LiAlSiO}_{4}, \mathrm{LiAlSi}_{5} \mathrm{O}_{12}\right.$ and $\mathrm{LiAlSi}_{10} \mathrm{O}_{22}$ ), regardless of the thermal pre-treatment provided, are sensitive to radiation. However, one can observe important differences in intensity, shape and peak positions in the glow curves.
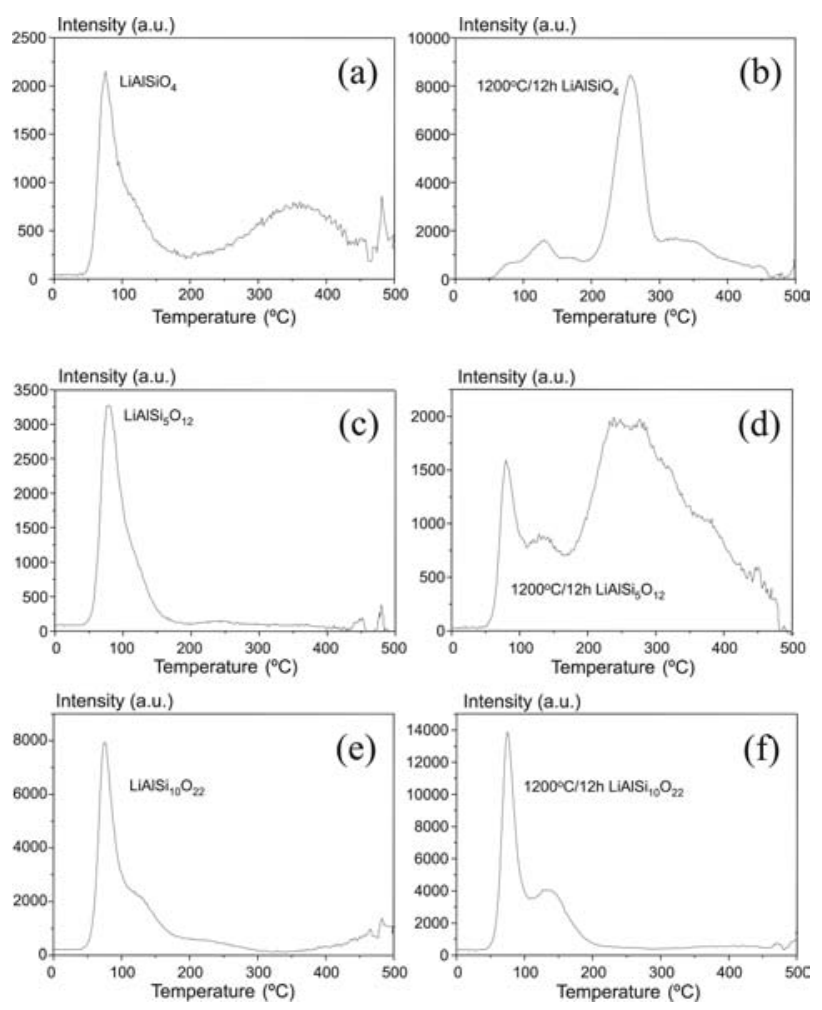

FIG. 2: TL glow curves of 5Gy irradiated synthetic (a,c,e) nonannealed and (b, d and f) $12 \mathrm{~h}$ at $1200^{\circ} \mathrm{C}$ thermally annealed lithiumrich aluminosilicates.

Clearly, the effect of a strong thermal treatment gives rise to an increase in the intensity of the TL signal when the tem- 
perature increases. According to Martini et al. [14], the electron-hole mechanisms explain the large UV-blue luminescence emissions $(380 \mathrm{~nm})$ from quartz-like structure materials. Prolonged high-temperature annealing of these compounds reduces the presence of ionic charge compensators $\left(\mathrm{H}^{+}, \mathrm{Na}^{+}, \mathrm{K}^{+}\right)$and sensitises the structure that increases its blue emissions during luminescence processes after irradiation $(\alpha, \beta, \gamma, U V, \mathrm{X}$-radiation, electron beam). It supposes that the electron-hole recombination is higher in thermal pretreatment samples than in non thermal pre-annealed ones. The $A l^{3+}$ ion is substituting $\mathrm{Si}^{4+}$ and for local charge compensation, a positive charge is needed (a trapped hole, $h^{+}$).

The more probable sequence of the process could be described as follows. Due to the exposure to beta radiation, the electrons are released from the oxygen atoms closed to the aluminum giving rise to electro-hole pairs. It should generate $\left[\mathrm{AlO}_{4} / h^{+}\right]$centers that deliver alkali $\left(\mathrm{Li}^{+}\right)$to diffuse away in the lattice. The alkali should join to an electron (e) to become neutral in the crystal network. Both the increase of the temperature that induces the migration of the electronhole pairs and, on the other hand, $\left[\mathrm{AlO}_{4} / h^{+}\right]$centers combined with electrons are the origin of the $\left[\mathrm{AlO}_{4}\right]^{\circ}$ centers. At the same time, these centers can be linked to Li or electronholes producing $\left[\mathrm{AlO}_{4} / \mathrm{Li}^{+}\right]$and $\left[\mathrm{AlO}_{4} / \mathrm{h}^{+}\right]$centers respectively. The production-annihilation of luminescent $\left[\mathrm{AlO}_{4}\right]^{\circ}$ centers could be analysed in terms of ionic self-diffusion. In this case, the combination of the thermal annealing and the subsequent beta irradiation should increase the ionicity of the $\mathrm{Si}-\mathrm{O}$ bonds modifying the ground state where each $\mathrm{Si}$ atom has four valence electrons and each $\mathrm{O}$ atom six valence electrons, implying that $\mathrm{O}$ states would exceed in number $\mathrm{Si}$ states by 3:1 considering atoms widely separated. The resulting centers are $\left[\mathrm{AlO}_{4} / \mathrm{Li}^{+}\right],\left[\mathrm{AlO}_{4} / \mathrm{h}^{+}\right]$and $\left[\mathrm{AlO}_{4}\right]^{o}$ that are coupled during the thermal self-diffusion processes through the bulk and interfaces. According to Itoh et al [15], the UVblue emission could be attributed to $\left[\mathrm{AlO}_{4} / h^{+}\right]$, (the transitions performed at 365 and $475 \mathrm{~nm})$, and $\left[\mathrm{AlO}_{4}\right]^{-}$centers (the $380 \mathrm{~nm}$ emission).

The variation in the shape and peak positions of the TL signal could be mainly a consequence of the strong thermal annealing given to the samples. During the annealing treatment there are different processes that simultaneously can occur in the material, (i) reversible phase transition from beta-quartz structure (hexagonal symmetry) to keatite (tetragonal symmetry) [1] or phase transformations [13]; (ii) consecutive breaking linking of bonds where thermal preheating reduces the adsorbed water content and the hydroxyl groups, $\equiv \mathrm{Si}-\mathrm{OH}+\mathrm{HO}-\mathrm{Si} \equiv \longrightarrow \equiv \mathrm{Si}-\mathrm{O}-\mathrm{Si} \equiv+\mathrm{H}_{2} \mathrm{O}$ [16]; (iii) alkali self-diffusion through the void channels in the aluminosilicate lattice [17] and (iv) redox reactions of the sort: $A l-O^{2-}-A l \rightarrow A l-O^{-}-A l+e^{-}$and the free electrons can be captured by $\left[\mathrm{SiO}_{3}\right]^{2+}$ producing $\left[\mathrm{SiO}_{3}\right]^{+}\left(E_{1}^{\prime}\right.$ like center). So $\left[\mathrm{SiO}_{3}\right]^{+}+e^{-} \rightarrow\left[\mathrm{SiO}_{3}\right]^{o}[18]$. One can distinguish this TL changes that are in agreement with the observed modifications in the X-ray diffractograms that show variations in the structure of the patterns.

Both annealed and non-annealed samples exhibit a basic common structure with an intense emission at $90^{\circ} \mathrm{C}$ except the annealed $\beta$-eucryptite with a very low intensity signal. The group of components that produce this peak has been
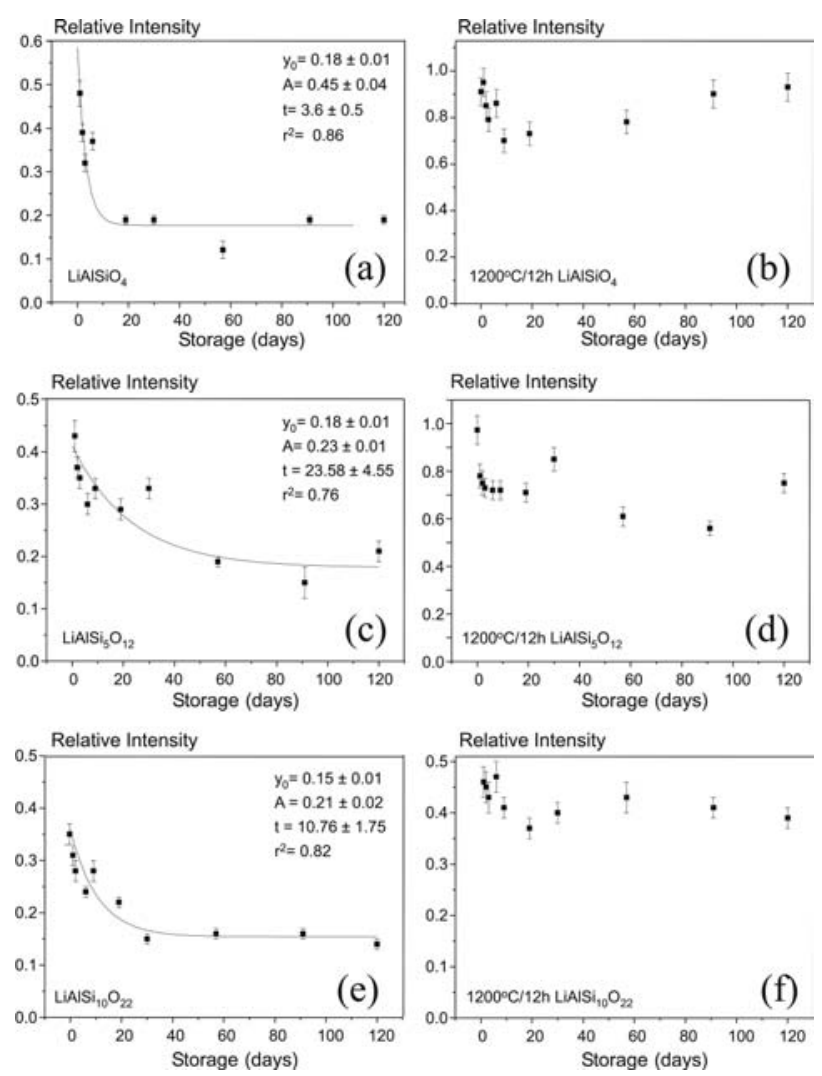

FIG. 3: Stability of the TL signal of 5Gy beta irradiated samples over 120 days stored at RT under red light.

also detected in the TL glow curves obtained for different irradiated natural quartzes [19] and aluminosilicates (e.g. microcline, a K-rich feldspar [20]), so it could be intrinsically associated with the $\mathrm{Si}-\mathrm{O}$ bonds in the lattice, regardless of the type and concentration of impurities and the lattice structure. Non-annealed samples (Fig 2a, 2c and 2e) show slight differences depending on the dopant concentration. The lowest Li-Al concentration sample displays three maxima peaked at $90^{\circ} \mathrm{C}, 130^{\circ} \mathrm{C}$ and $200^{\circ} \mathrm{C}$ (Fig 2e). When the dopant level is increased $\left(\mathrm{LiAlSi}_{5} \mathrm{O}_{12}\right)$, the intensity of the $90^{\circ} \mathrm{C}$ TL emission decreases (more than twice) and the peaks at $130^{\circ} \mathrm{C}$ and $200^{\circ} \mathrm{C}$ change not only the shapes but also they move their positions. Thus, in Fig $2 \mathrm{a}$ and $2 \mathrm{c}$ the $130^{\circ} \mathrm{C}$ peak is not so easily detectable and the $200^{\circ} \mathrm{C}$ peak is shifted to about $240^{\circ} \mathrm{C}$ (Fig 2c) and cannot be detectable for the $\mathrm{LiAlSiO}_{4}$ (Fig 2a). The intensity of the $130^{\circ} \mathrm{C}$ peak in $\beta$-eucryptite appears with ratios of 7:2 respect to $\mathrm{LiAlSi}_{10} \mathrm{O}_{22}$ and 3:2 respect to virgilite-petalite crystals. However, the peculiarity of the $\beta$-eucryptite lies in the appeareance of a wide maximum peaked at $350^{\circ} \mathrm{C}$, in a position corresponding to the 'empty' zone of the others samples (Fig 2a). The changes in the TL intensity indicate that the Li-Al dopants act as TL inhibitors giving rise non-radiative $\left[\mathrm{AlO}_{4} / \mathrm{Li}^{+}\right]$centers that are able to compete with the holes hosted in luminescent centers. The higher is the Li-Al dopant content, the lower is the intensity of the TL glow curves in non-annealed samples. The shift of the $200^{\circ} \mathrm{C}$ maxima toward higher temperatures indicates that higher dopant leveles suppose progressive creation of deeper energy traps which need to be emptied at higher 
temperatures.

Thermal annealing on the LAS species induces drastic changes in the shape of the TL emission of 5Gy irradiated $\beta$ eucryptite (Fig 2b) and virgilite-petalite (Fig 2d) crystals, but is scarcely significant for $\mathrm{LiAlSi}_{10} \mathrm{O}_{22}$ (Fig 2f), when compared to non-annealed samples. The almost negligible differences observed in Fig $2 \mathrm{f}$ respect to Fig 2e are related to: (i) the intensity of the $90^{\circ} \mathrm{C}$ TL emission (ratio of $4: 7$ respect to the non-annealed $\mathrm{LiAlSi}_{10} \mathrm{O}_{22}$ ), (ii) the slight shift (about $10^{\circ} \mathrm{C}$ ) of the second maximum that is peaked at $140^{\circ} \mathrm{C}$ and (iii) the non-detected group of components over $200^{\circ} \mathrm{C}$ for annealed samples and $300^{\circ} \mathrm{C}$ in non-annealed ones.

TL curves in Figs $2 b$ and $2 d$ share, among them, the different behaviour when compared respectively to the nonannealed $\mathrm{LiAlSiO}_{4}$ and $\mathrm{LiAlSi}_{10} \mathrm{O}_{22}$. Both exhibit a very complex TL structure with not well-defined group of components. One can guess two peaks at $90^{\circ} \mathrm{C}$ and $140^{\circ} \mathrm{C}$ that are in common with the non-annealed samples, but the intensities are quite different. In fact, these are not the main (i.e. the more intense) maxima in the curve. This role corresponds to the group of components appearing over $200^{\circ} \mathrm{C}$ that are almost negligible in non annealed samples. Clearly the appeareance of this signal is directly linked to the strong thermal treatment; and it is obvious that higher concentration of dopants induces an increase in the thermal sensitivity probably associated with alkali self-diffusion processes, phase transformations and generation of glassy phases. The presence of glassy materials affects to the TL curve shape giving rise to emission with not well-defined maxima. The sample with lower levels of $\mathrm{Li}$ and $\mathrm{Al}\left(\mathrm{LiAlSi}_{10} \mathrm{O}_{22}\right.$ with quartz structure) exhibits a higher strength in the stability of the lattice structure when applied severe thermal treatments.

\subsection{Fading effect}

Laboratory driven thermoluminescence tests were performed on non-annealed and preannealed $\mathrm{LiAlSiO}_{4}$, $\mathrm{LiAlSi}_{5} \mathrm{O}_{12}$ and $\mathrm{LiAlSi}_{10} \mathrm{O}_{22}$, up to $1200^{\circ} \mathrm{C}$ for $12 \mathrm{~h}$ to check the temporal evolution of the TL blue signal after 5Gy of beta irradiation. Assuming that the TL sensitivity of the aliquots could be affected by white light, all the samples were stored under red light at RT to prevent trapped electron releasing from semi-stable sites into hole centres, including luminescence centres. Measurements were made after increasing the storage periods of time (up to 120 days) using four replicates each. The calculation of each point was calibrated individually for each aliquot, referring the direct delayed measurement (area of the glow curve in a range of $50^{\circ}-500^{\circ} \mathrm{C}$ ) to a $5 \mathrm{~Gy}$ induced prompt TL glow curve, in the same range of temperature, avoiding the influence of the weight factor. The uncertainties $(1 \sigma)$ display the precision of the mean value (MV) of the luminescence response (counts in arbitrary units) corresponding to the group of four aliquots.

As shown in Figure 3 (left-hand plots corresponding to non-annealed Li-rich aluminosilicates), the temporal evolution of the TL emission of this material is close to other materials previously studied [20] starting with an initial rapid decay (ca. 75-80\%) and maintaining stability from about 30 days onwards. The TL signal behaviour is associated with the probability of electron release from the shallower traps that occurs very fast at RT. About three-four weeks later, the electron population decreases asymptotically by the X-axis and the involved electrons are located in deeper traps; consequently more energy is needed to leave their positions at RT. The number of trapped electrons depends on the time that the pre-annealed lithium aluminosilicate lattice needs to reach up the stabilisation after receiving the beta irradiation treatment. That is to say, the time necessary for the recombination of all the electrons that are released in the material from the $\mathrm{Al}-\mathrm{O}^{2-} \mathrm{Al}$ centres. As aforementioned, one can consider that these electrons are captured by $\left[\mathrm{SiO}_{3}\right]^{2+}$ giving rise to $\left[\mathrm{SiO}_{3}\right]^{+}\left(E_{1}^{\prime}\right.$-like centre $)[18]$.

This physical process, observed in non-annealed samples, can be fitted to a first order decay equation of the form $y=y_{0}+A \exp (-x / t)$ where: $y$ corresponds to the relative intensity of the TL signal, $x$ is the elapsed time after irradiation process and $y_{0}, A$ and $\mathrm{t}$ are coefficients of the equation. An acceptable value of the regression coefficients of fitting (never lower than $r=0.87$ ) was obtained. Although a nonexponential decay for first order kinetics may be expected as a consequence of the energy distribution of trapped charges [3], the results at room temperature can be well-fitted by a single exponential component. This is not surprising because at this temperature only the lowest energy traps of the exponential distribution is involved and they can be effectively considered as a single discrete trap at energy $E_{0}$. On the contrary, the evolution of the TL signals of annealed samples with the elapsed time shows an important scattering being impossible the fitting to an exponential decay (right-hand plots of Fig 3).

\section{CONCLUSIONS}

Thermoluminescence emission of different members of LAS $\left(\mathrm{LiO}_{2}-\mathrm{Al}_{2} \mathrm{O}_{3}-\mathrm{SiO}_{2}\right)$ series (i) $\beta$-eucryptite $\left(\mathrm{LiAlSiO}_{4}\right)$, (ii) virgilite-petalite $\left(\mathrm{LiAlSi}_{5} \mathrm{O}_{12}\right)$ and (iii) virgilite-petalite-bikitaite $\left(\mathrm{LiAlSi}_{10} \mathrm{O}_{22}\right)$ have been scarcely investigated. The observed variation in the blue TL glow curves of different beta irradiated $\mathrm{LiAlSiO}_{4}, \mathrm{LiAlSi}_{5} \mathrm{O}_{12}$ and $\mathrm{LiAlSi}_{10} \mathrm{O}_{22}$ after a strong thermal annealing treatments could be linked to: (i) reversible phase transitions, (ii) consecutive breaking linking of bonds, (iii) alkali self-diffusion through the void channels in the aluminosilicate lattice and/or (iv) redox reactions. The stability of the TL signal after four months of storage performed at RT under red light, shows noteworthy differences between annealed ( 12 hours at $1200^{\circ} \mathrm{C}$ ) and non-annealed samples. Just like to other phosphors, the fading process in non-annealed samples can be fitted to a first-order decay mathematical expression; however preheated samples could not be reasonably fitted due to the high scattering detected. The more significant changes observed in the X-ray diffractograms are in the intensity of the peaks that denote modifications in the degree of crystallinity and in the appeareance of new peaks that denotes new phases as a consequence of the thermal annealing treatment. Despite the obtained results do not confirm the utility of these Li-rich aluminosilicates in accidental dosimetry, it could be 
considered as a good starting point for its potential use in this field. These materials exhibit similar luminescence properties to other alkali-rich aluminosilicates employed for dosimetric purposes, since display (i) an acceptable sensitivity to radiation, (ii) UV-blue TL emission, and (iii) a reasonable stability of TL signal (low fading) after 120 days of storage for non-annealed samples. Nevertheless, further works are necessary to to complete the dosimetric characterization of these materials.

\section{Acknowledgements}

This work has been funded by the CICYT (FIS200761823) projects. The authors thank Dr. Sanchez-Muñoz for supplying the material.
[1] M. Guedes, A.C. Ferro and J.M.F. Ferreira, J. Eur Ceram Soc 21(9) 1187 (2001)

[2] S. W. S. McKeever, Thermoluminescence of solids. (Cambridge Univ. Press, New York) 1985, p. 122.

[3] J.M. Gomez-Ros, V. Correcher, J. Garcia-Guinea and A. Delgado, Radiat. Prot. Dosim. 100, 399 (2002).

[4] V. Correcher, J.M. Gomez-Ros, J. Garcia-Guinea and E. Crespo-Feo., Mater. Chem. Phys. 116(1) 143 (2009).

[5] V. Correcher, L. Sanchez-Munoz, J. Garcia-Guinea, J.M. Gomez-Ros and A. Delgado, Nucl. Instrum. Meth. A. 562(1) 456 (2006).

[6] J.M. Gomez-Ros, V. Correcher, L. Sanchez-Munoz, J. GarciaGuinea and A. Delgado, Phys. Status Solidi A 204(3) 861 (2007).

[7] V. Correcher, J. Garcia-Guinea, L. Sanchez-Munoz and A. Delgado, J. Mater. Process. Tech. 143871 (2003a).

[8] D.L. Hamilton and C.M.B. Henderson, Miner. Magaz. 36832 (1968).

[9] J. Daniel-Martin, X-powder, a software package for powder X-ray diffraction analysis. Version 2004.03. URL: www.xpowder.com. (2004).

[10] L. B $\varnothing$ tter-Jensen and G.A.T. Duller, Nucl. Tracks Radiat.
Meas. Part D. 20549 (1992)

[11] V. Correcher and A. Delgado, Radiat. Meas. 29(3-4) 411 (1998).

[12] J. Garcia-Guinea and V. Correcher, Spectrosc. Lett. 33103 (2000).

[13] A.A. Dymnikov, O.S. Dymshits, A.A. Zhilin, V.A. Savostjanov and T.I. Chuvaeva, J. Non-Cryst Solids. 19667 (1996)

[14] M. Martini, A. Paleari, G. Spinolo and A. Vedda, Phys Rev. B 52(1) 138 (1995).

[15] N. Itoh, D. Stoneham and A.M. Stoneham, J. Appl. Phys. 92(9) 5036 (2002)

[16] L.L. Stillings, S.L. Brantley and M.L. Machesky, Geochim. Cosmochim Acta 59(8) 1473 (1995)

[17] V. Correcher, J. Garcia-Guinea and A. Delgado, Radiat. Meas. 32(5-6) 709 (2000)

[18] S.O. Souza, J.F.D. Chubaci, P.C. Selvin, M.D. Sastry and S. Watanabe, J. Phys. D-Appl Phys 35(13) 1562 (2002).

[19] V. Correcher, J. Garcia-Guinea, A. Delgado and L. SanchezMunoz, Radiat. Prot. Dosim. 84(1-4) 503 (1999)

[20] V. Correcher, J. Garcia-Guinea and F.J. Valle-Fuentes, Geophys. Res. Lett. 1949 10.1029/2003GL018028 (2003b) 